\title{
Phase behavior and rheological characterization of silica nanoparticle gel
}

\author{
Cigdem O. Metin • Kelli M. Rankin • \\ Quoc P. Nguyen
}

Received: 27 September 2012/ Accepted: 12 October 2012/Published online: 30 October 2012

(C) The Author(s) 2012. This article is published with open access at Springerlink.com

\begin{abstract}
Preferential injection into high permeability thief zones or fractures can result in early breakthrough at production wells and large unswept areas of high oil saturation, which impact the economic life of a well. A variety of conformance control techniques, including polymer and silica gel treatments, have been designed to block flow through the swept zones. Over a certain range of salinities, silica nanoparticle suspensions form a gel in bulk phase behavior tests. These gels have potential for in situ flow diversion, but in situ flow tests are required to determine their applicability. To determine the appropriate scope of the in situ tests, it is necessary to obtain an accurate description of nanoparticle phase behavior and gel rheology. In this paper, the equilibrium phase behavior of silica nanoparticle solutions in the presence of sodium chloride $(\mathrm{NaCl})$ is presented with four phase regions classified as a function of salinity and nanoparticle concentration. Once the gelation window was clearly defined, rheology experiments of silica nanoparticle gels were also carried out. Gelation time decreases exponentially as a function of silica concentration, salinity, and temperature. Following a power law behavior, the storage modulus, $G^{\prime}$, increases with particle concentration. Steady shear measurements show that silica nanoparticle gels exhibit nonNewtonian, shear thinning behavior. This comprehensive study of the silica nanoparticle gels has provided a clear
\end{abstract}

C. O. Metin · K. M. Rankin · Q. P. Nguyen $(\bowtie)$

Department of Petroleum and Geosystems Engineering,

The University of Texas at Austin, CPE 5.104A,

200 E. Dean Keeton, Stop C0300, Austin, TX 78712, USA

e-mail: quoc_p_nguyen@mail.utexas.edu path forward for in situ tests to determine the gel's applicability for conformance control operations.

Keywords Silica nanoparticle $\cdot$ Gel $\cdot$ Phase behavior . Rheology · Conformance control

\section{Introduction}

As discussed by Metin et al. (2011), in the presence of electrolytes, nanoparticle dispersions become unstable above a critical salt concentration. Over a certain range of salinities, the unstable solutions form a gel. Previous conformance control efforts with silica gels (e.g., Jurinak and Summers 1991; Burns and al 2008; Dai et al. 2010; Stavland et al. 2011) focused on the gelation of sodium silicate solutions triggered by $\mathrm{pH}$ or salinity changes prior to injection and required high silica concentrations. Conformance control operations using the silica nanoparticle gels discussed in this paper have three key differences from previously studied silica gels: the silica is in the form of colloidal silica, a suspension of fine amorphous, nonporous, and typically spherical silica particles $\left(\mathrm{SiO}_{2}\right)$ in the liquid phase, not sodium silicate powder $\left(\mathrm{Na}_{2} \mathrm{SiO}_{3}\right)$; the gelation would be controlled by salinity contrasts within the reservoir rather than being triggered by changes in $\mathrm{pH}$ or salinity prior to injection; and gelation occurs at very low silica concentrations.

This paper presents the equilibrium phase behavior of silica nanoparticle dispersions in the presence of sodium chloride $(\mathrm{NaCl})$ and the rheology of particle dispersions in the gel region. The characteristic time for the onset of gelation as a function of silica and $\mathrm{NaCl}$ concentration and temperature was also determined.

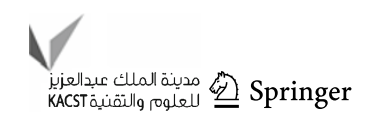




\section{Materials and methods}

The material under study is aqueous dispersions of silica nanoparticles. The nanoparticles provided by $3 \mathrm{M}$, Co. (St. Paul, MN) have a mean diameter of $5 \mathrm{~nm}$. The NexSil5 nanoparticles purchased from Nyacol have a bimodal size distribution with peaks at 3 and $18 \mathrm{~nm}$. Phase behavior tests were carried out in glass vials in temperature-controlled ovens. Phase behavior diagrams were constructed at $25{ }^{\circ} \mathrm{C}$ for both particles and at 70 and $90{ }^{\circ} \mathrm{C}$ for the NexSil5 particles.

Rheological measurements were carried out on an ARES LS-1 rheometer using a parallel plate fixture. The diameter of the parallel plate was $50 \mathrm{~mm}$ and the set gap between the lower and upper plate was $1 \mathrm{~mm}$. The measurements were conducted at $25,40,55$ and $75{ }^{\circ} \mathrm{C}$. A solvent trap was used to prevent the evaporation of water from the solution. Dynamic and steady shear experiments were carried out. The nanoparticle dispersion was mixed with the $\mathrm{NaCl}$ solution and then placed on the lower fixture of the parallel plate. A dynamic time sweep test was first conducted at $1 \mathrm{rad} / \mathrm{s}$ frequency and $1 \%$ strain. The time evolution of storage and loss modulus, $G^{\prime}$ and $G^{\prime \prime}$, was measured for $10,000 \mathrm{~s}$ after the onset of gelation. Then, frequency sweep and strain sweep tests were carried out. After these tests, steady shear rates were applied and viscosity was recorded as a function of shear rate.

\section{Results and discussion}

The results on phase behavior of silica nanoparticle suspensions as a function of $\mathrm{NaCl}$ and nanoparticle concentration are presented in this section. The rheological measurements of samples in the gel region are discussed in detail in the next section.

\section{Phase behavior}

There are three phases in the nanoparticle phase diagram. Below the critical salt concentration (CSC) (Metin et al. 2011), the nanoparticle dispersion is stable and appears as a homogeneous clear liquid. Above the CSC, the suspension is unstable. The unstable suspensions have two distinct behaviors. At salinities above the CSC but below the upper gelation salt concentration, the unstable solution forms a gel phase. Many authors have proposed that gel formation is due to dynamic arrest of the particle clusters (Campbell et al. 2005; Lu et al. 2008; de Candia et al. 2005). In colloidal suspensions, for longer separation lengths, repulsion is greater than Van der Waals attraction resulting in a long range repulsive barrier. At shorter lengths, Van der Waals attraction is still the dominating force (de Candia et al.

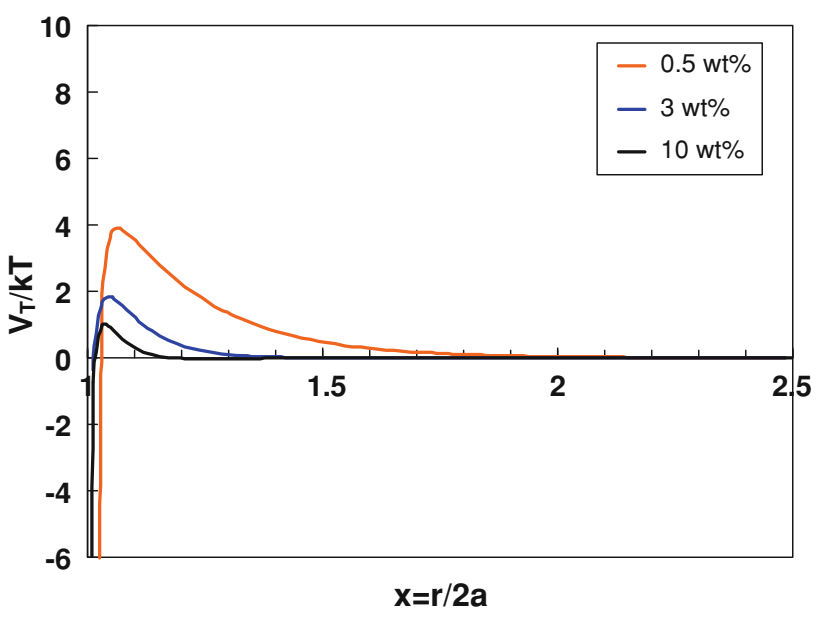

Fig. 1 Total interaction potential for $5 \mathrm{~nm} 3 \mathrm{M}$ silica nanoparticles for $0.5,3$ and $10 \mathrm{wt} \% \mathrm{NaCl}$

2005). The short range attraction is responsible for cluster growth. The long range repulsive forces lock the clusters into a cage (gel) during dynamic arrest (Campbell and al 2005). Above the upper gelation salt concentration, the solution forms a viscous liquid. The major visual difference between the two phases is that the gel does not flow when the sample is tilted, but the viscous liquid does flow. In the viscous liquid region, we believe that the network of aggregates, which is essential for gel formation, is absent. This is supported by the theory of dynamic arrest, which requires long range repulsive forces to suspend the clusters in a gel. Without a repulsive force, cluster growth continues which produces a viscous fluid but no gel properties. Figure 1 shows the total interaction potential between $5 \mathrm{~nm}$ diameter nanoparticles in aqueous dispersion at increasing salinities. As the salt concentration increases, the repulsive energy barrier decreases. The transition between gel and viscous liquid occurs when the repulsive force is no longer sufficient to initiate dynamic arrest.

It is known that strongly aggregating colloidal suspensions form gels, whose stability depends on the volume fraction of the particles (Senis and Allain 1997). Within the gel region, two types of gels are observed. At lower silica concentrations, the solution has two phases. A solid gel phase is topped by a clear supernatant liquid. At higher silica concentrations, the solution is a single-phase gel. Two-phase gels are a result of insufficient number of aggregates to incorporate the entire volume of water into the gel. Figures 2 and 3 show phase behavior diagrams for the $3 \mathrm{M}$ and NexSil5 particles, respectively, at $25^{\circ} \mathrm{C}$. The monodisperse $3 \mathrm{M}$ particles have a wider gel region with respect to salinity than the bimodal NexSil5 particles. The boundary between single-phase and two-phase gel is only a function of silica concentration for both particles. 


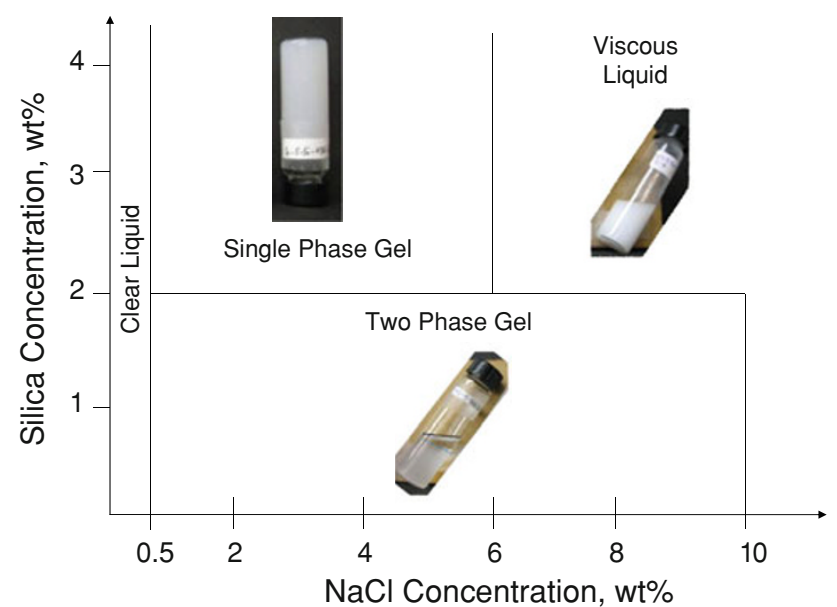

Fig. 2 Phase behavior diagram of $5 \mathrm{~nm} 3 \mathrm{M}$ silica nanoparticles at $25{ }^{\circ} \mathrm{C}$

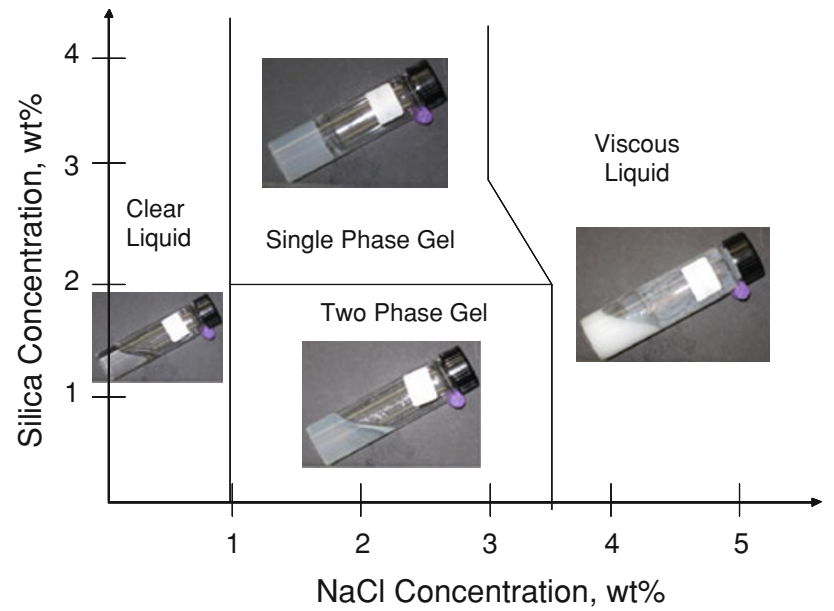

Fig. 3 Phase behavior diagram of NexSil5 silica nanoparticles at $25{ }^{\circ} \mathrm{C}$

When the particle volume fraction is small, isolated large flocs, which are denser than the suspending medium, may form and can sediment under gravity. However, for large particle volume fraction, gelation usually occurs and sedimentation is then avoided unless the gel structure is so fragile that it collapses under its own weight (Larson 1999). The sedimentation phase was not observed in our gel region suggesting that the critical volume fraction for gelation must be less than $1 \mathrm{wt} \%$ (the lowest value studied). Figure 4 is a schematic presentation of sedimentation and gelation of aggregates. The structure of the aggregates within the sediment, such as the solid volume fraction, is discussed based on the equilibrium approach by Metin et al. (2012a). Even though both nanoparticle dispersions display the same four phase behavior regions, the extent of each region, specifically the gelation window, is different. As a consequence, the range of reservoir salinities for which each particle will gel in situ will change depending

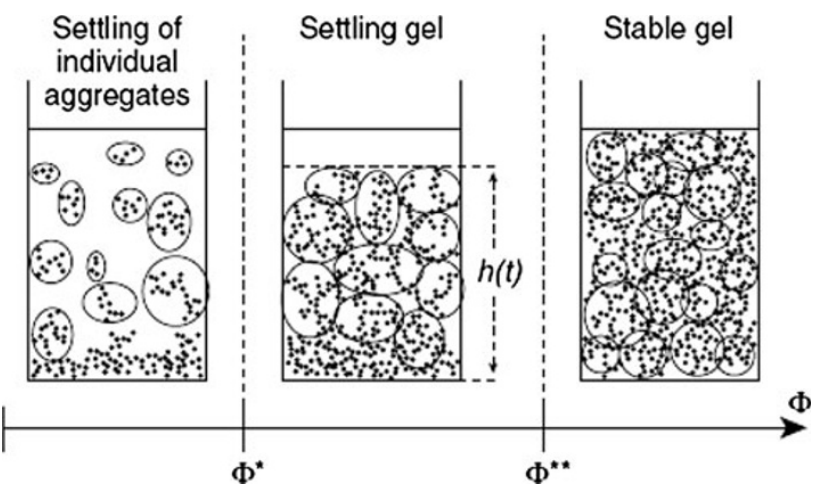

Fig. 4 Schematic presentation of sedimentation and gel behavior of aggregates as proposed by Senis and Allain (1997)

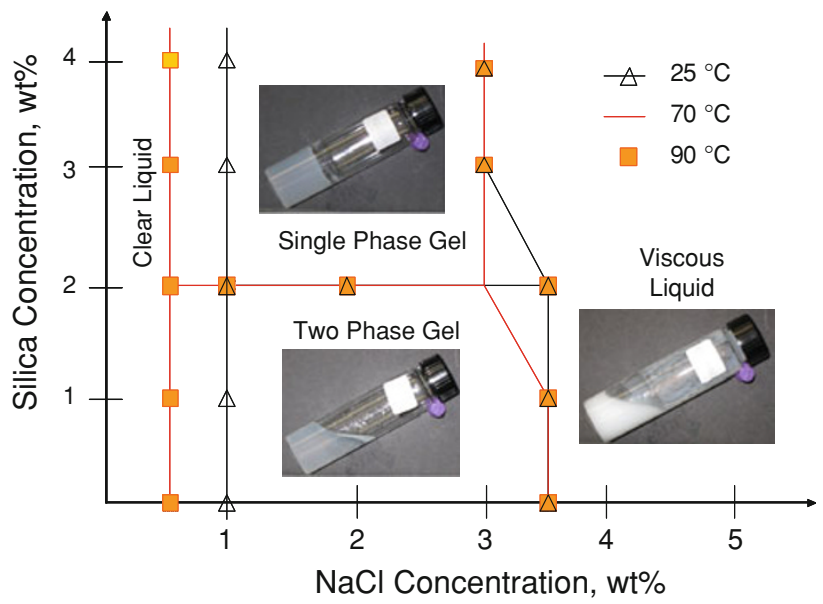

Fig. 5 The impact of temperature on the phase behavior diagram of the NexSil5 silica nanoparticle dispersion

on which particle is used. Our work suggests that the phase behavior of any given nanoparticle will have to be clearly understood before application to ensure that gelation will occur at reservoir salinity.

The effect of temperature on particle phase behavior was studied for the NexSil5 particles. Figure 5 shows the phase diagrams for the NexSil5 particle at 25, 70, and $90{ }^{\circ} \mathrm{C}$. The primary impact of increasing temperature is to decrease the critical salt concentration. This observation may be explained based on relative magnitude of the energy barrier and the average kinetic energy of nanoparticles. An increase of the average kinetic energy with temperature gives rise to the particle collisions that result in aggregation. As a consequence, a higher energy barrier (i.e., lower salt concentration) is required to maintain the aqueous stability of the nanoparticle dispersion.

Rheological measurements

In addition to understand the equilibrium behavior of the solutions to correctly identify the gelation window, it is 
important to understand the kinetics of the gelation. A way to explore rates of structural rearrangement within a complex fluid without significantly deforming the fluid's microstructure is to apply small-amplitude oscillatory shearing (Larson 1999). The sinusoidally varying stress can be represented as

$\sigma(t)=\gamma_{0}\left[G^{\prime}(\omega) \sin (\omega t)+G^{\prime \prime}(\omega) \cos (\omega t)\right]$

Shear stress $\omega(t)$ is proportional to the amplitude of the strain $\gamma_{0}$. In Eq. 1, $G^{\prime}(\omega)$ is the storage modulus and is in phase with the strain. $G^{\prime \prime}(\omega)$ is the loss modulus and is in phase with the rate of strain. The storage modulus represents the storage of elastic energy, and the loss modulus represents the viscous dissipation of that energy (Larson 1999). The ratio $G^{\prime \prime} / G^{\prime} \gg 1$ represents materials that are liquid-like, and the ratio $G^{\prime \prime} / G^{\prime} \ll 1$ represents solid-like materials.

Early on, aggregate size grows exponentially in time until the fractal aggregates become large enough to form a network and fill the entire volume of the solution if nanoparticle concentration is sufficient. The system then crosses over to the critical growth associated with gelation (Martin and Wilcoxon 1989). Measurements taken with dynamic light scattering (DLS) and ultraviolet-visible spectrophotometer (UV-vis) confirm the exponential growth of aggregate size in time before the onset of gelation. The UV-vis measurements were used as a gelation time estimate. For samples with gelation times in excess of a few hours, the samples were mixed and allowed to sit in closed vials prior to placing the solution on the rheometer plate to reduce measurement time prior to gelation.

Dynamic time sweep tests were conducted to quantify the gelation time as a function of silica concentration, salinity, and temperature. Salinity and temperature are reservoir properties that are constraints to the gel's application for conformance control. Silica concentration will be an operating variable that can be adjusted as necessary to ensure gelation at in situ conditions and control project economics. The concentrations for the silica and salinity scan were chosen so that they would fall within the gelation window of both particles. A secondary silica scan was run for the $3 \mathrm{M}$ particle at a higher salinity outside of the NexSil5 gelation window. For the primary silica scan, the temperature was constant at $25{ }^{\circ} \mathrm{C}$ with a salinity of 3 wt $\% \mathrm{NaCl}$. For the secondary silica scan, the temperature was constant $25^{\circ} \mathrm{C}$ with a salinity of 5 wt $\% \mathrm{NaCl}$. For the salinity scan, the temperature was constant at $25{ }^{\circ} \mathrm{C}$ with a silica concentration of $4 \mathrm{wt} \%$. For the temperature scan, the silica concentration was $3 \mathrm{wt} \%$ with a salinity of 3 wt $\%$. The scans are overlain on the phase diagram of the 3M nanoparticle in Fig. 6.

The onset of gelation is determined by a UV-vis spectrophotometer and strain-controlled rheometer. The

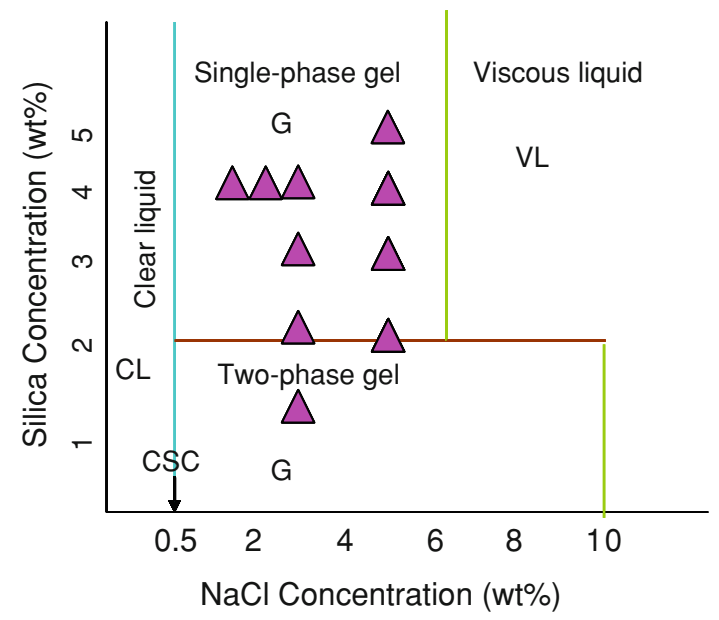

Fig. 6 Schematic presentation of the samples studied during rheology experiments

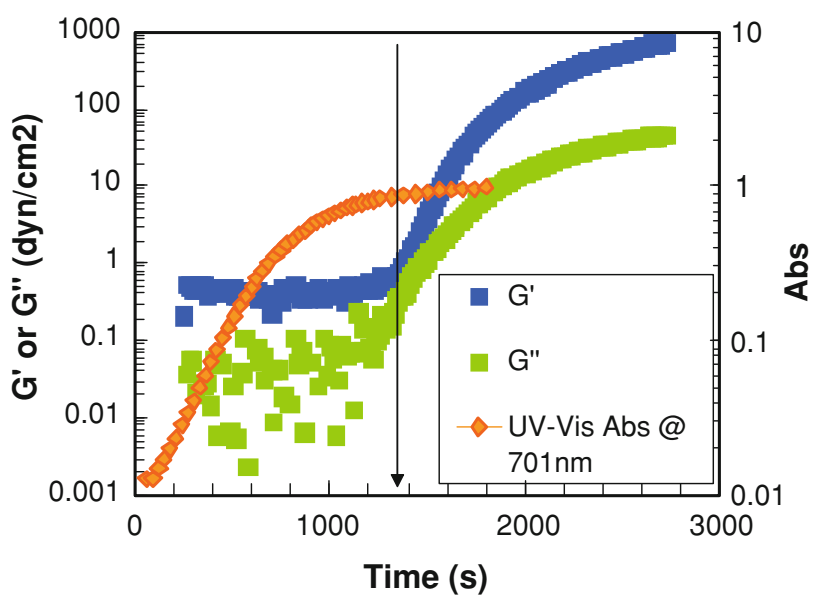

Fig. 7 Dynamic time sweep test and UV-vis absorbance of 4 wt \% $3 \mathrm{M}$ silica and 5 wt $\% \mathrm{NaCl}$

evolution of $G^{\prime}$ and $G^{\prime \prime}$ is recorded as a function of time and the onset of gelation is determined based on the sudden increase of $G^{\prime}$, as shown with an arrow in Fig. 7. The onset of gelation, estimated by evaluating the sudden change in $G^{\prime}$ in the rheology experiments, agrees well with that determined by the change in absorbance at a specific wavelength (700 $\mathrm{nm}$ in this case) as measured using a UVvis spectrophotometer. A change in aggregate size can be captured by measuring the absorbance using a UV-vis spectrophotometer (Metin et al. 2011).

In Fig. 7, the increase in absorbance corresponds to the increase in aggregate size, and when the network is formed, a plateau is reached. The transition corresponds to the onset of gelation as determined by $G^{\prime}$ in rheology experiments. After the gelation time, $G^{\prime}$ increases significantly, while the ratio $G^{\prime \prime} / G^{\prime}$ becomes much smaller than one indicating that the sample has a solid-like behavior. The scattered data for early $G^{\prime} / G^{\prime \prime}$ measurements are due to a lack of accuracy at smaller 
values. The storage modulus increases during the dynamic measurements suggesting that the gel network gets stronger and stronger over time. Manley et al. (2005) also showed that colloidal silica gels stiffen with time through light scattering and rheological measurements. The authors argued that there was no change in the gel structure but the interparticle spring constant was time-dependent and responsible for the increase in $G^{\prime}$. According to their hypotheses, the kinetics of bond formation is reaction limited, which leads to an increase in the contact area between network forming particles.

Gelation time decreases as silica concentration increases as shown in Fig. 8. This observation is in agreement with the way in which the kinetics of the aggregation of silica nanoparticle suspensions change as a function of silica, as discussed by Metin et al. (2012b). For the 3M particles, the change in gelation time is more significant at $3 \mathrm{wt} \% \mathrm{NaCl}$ than it is at $5 \mathrm{wt} \% \mathrm{NaCl}$ (Fig. 8). The effect of particle size distribution on gelation time at constant salinity can also be seen in Fig. 8. The polydispersity seems to decrease the gelation time but the rate of decrease in gelation time as nanoparticle concentration increases is the same as that for monodisperse $5 \mathrm{~nm} 3 \mathrm{M}$ particles.

An increase in salinity decreases the gelation time (see Fig. 9). This observation is also in close agreement with the way in which the kinetics of aggregation of silica nanoparticle suspensions change, as reported by Metin et al. (2012b). The gelation time changes orders of magnitude as the $\mathrm{NaCl}$ concentration is increased. Again, the polydisperse NexSil5 particles have shorter gelation times, but for a given silica concentration show more significant decrease in gelation time with increasing salinity than for the monodisperse $3 \mathrm{M}$ particles (compare the slopes in Fig. 9).

Temperature also significantly effects the gelation time. An increase in temperature decreases the gelation time (see Fig. 10). The activation energy $\left(E_{a}\right)$ for kinetically

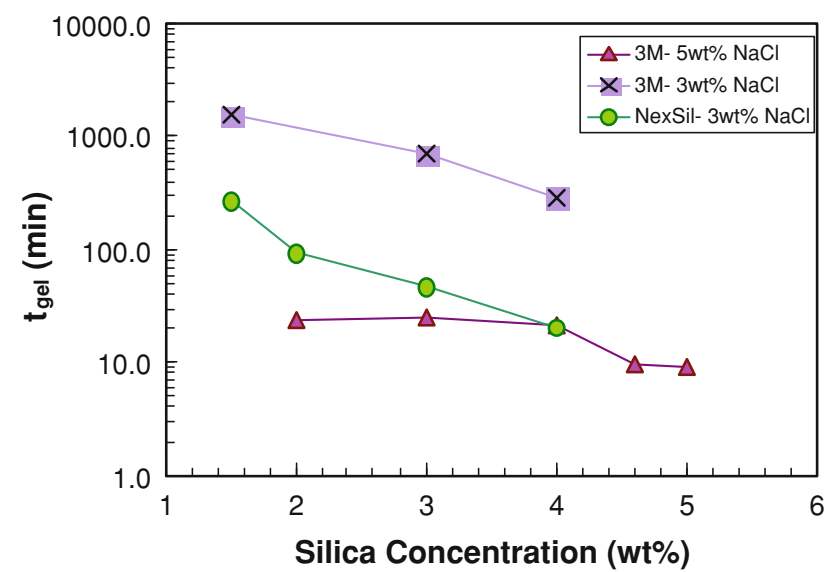

Fig. 8 Rheometer measurement of gelation time as a function of silica concentration at $25{ }^{\circ} \mathrm{C}$

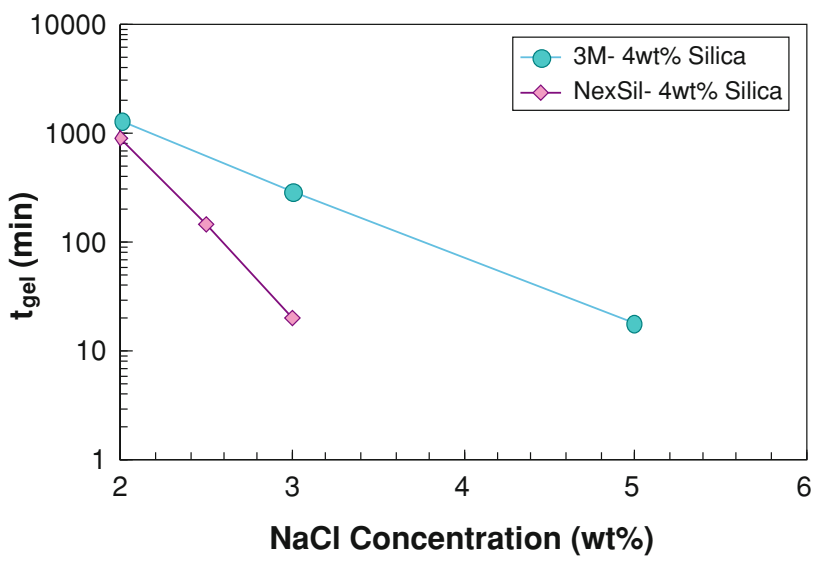

Fig. 9 Rheometer measurement of gelation time as a function of salinity for 4 wt $\%$ silica suspensions at $25{ }^{\circ} \mathrm{C}$

controlled cross-linking was used by Amiri et al. (2011) to characterize the temperature dependency of the gelation time for silica suspensions (Eq. 2).

$\ln t_{g}=\frac{E_{a}}{R T}+A$

where $t_{g}$ is the gelation time, $R$ is the gas constant, $T$ is the temperature in Kelvin, and $A$ is a constant. The activation energy is calculated using data in Fig. 10 to be 84 and $73 \mathrm{~kJ} / \mathrm{mol}$ for monodisperse $3 \mathrm{M}$ particles and polydisperse NexSil5 particles, respectively. These apparent activation energies for bridging of aggregates are comparable to the values reported by Silva and Vasconcelos (1999); Wang and Zhang (2009) and Amiri et al. (2011). The decrease in gelation time at high temperature was attributed to larger Brownian motion of the particles resulting in faster collisions (Metin et al. 2011). Similar results for gelation time were reported for silica particles suspended in ethanol by Smith and Zukoski (2006).

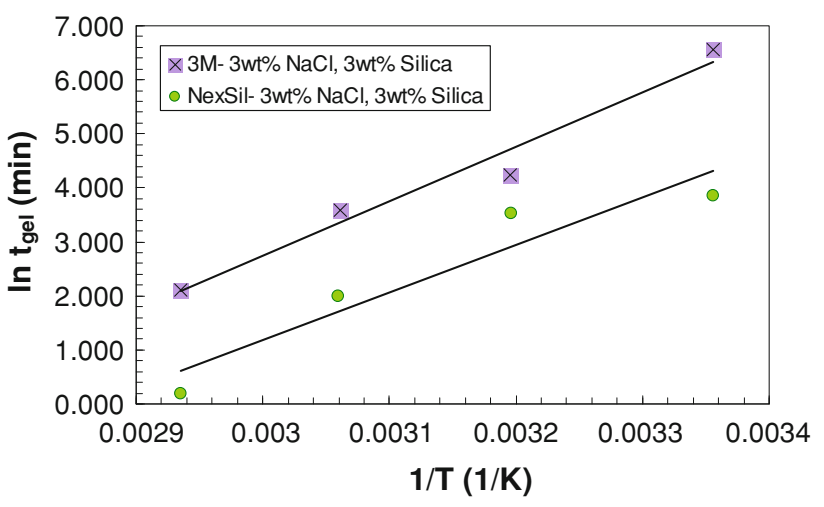

Fig. 10 Rheometer measurements of gelation time as a function of temperature for $3 \mathrm{wt} \%$ silica suspensions with $3 \mathrm{wt} \% \mathrm{NaCl}$. The lines correspond to theory given in Eq. 2 
The dependence of gelation time on silica, $\mathrm{NaCl}$ concentration, or temperature follows an exponential decrease (Figs. 8, 9 and 10). This means that a small change in any of the variables can result in a significant change in gelation time. The gelation time can vary an order of magnitude with changes in the primary control variables. This necessitates a thorough understanding of the gelation kinetics of the nanoparticles before designing a conformance control test. Other rheological quantities that are important for strongly flocculated gels include linear and nonlinear storage moduli at high and low frequencies and shear rate dependent viscosity (Larson 1999). The silica nanoparticle gels show solid-like behavior which is shown by the storage and loss modulus profiles in Figs. 11, 12, 13 and 14. Hyun et al. (2002) used large amplitude oscillatory shear (LAOS) behavior to classify polymer samples. We applied this classification to the nanoparticle samples. The decrease in $G^{\prime \prime}$ at low shear rates was not observed by Hyun et al. (2002), so only the $G^{\prime \prime}$ behavior after the minimum was used for interpretation. For most samples studied the $G^{\prime}$ and $G^{\prime \prime}$ profiles show an increase followed by a decrease as strain increases, which is classified as strong strain overshoot. For the $G^{\prime}$ profiles (Figs. 11 and 13), the increase is difficult to see because of the large range on the $y$ axis.

Strain hardening arises from strong secondary bonding effects, such as the formation of a shear induced network. Initial increase in loss modulus with strain is attributed to structural changes during the breakdown of agglomerates to a larger number of smaller size units which are more
Fig. 11 Storage modulus $\left(G^{\prime}\right)$ as a function of silica concentration at $25{ }^{\circ} \mathrm{C}$
Fig. 12 Loss modulus $\left(G^{\prime \prime}\right)$ as a function of silica concentration at $25^{\circ} \mathrm{C}$
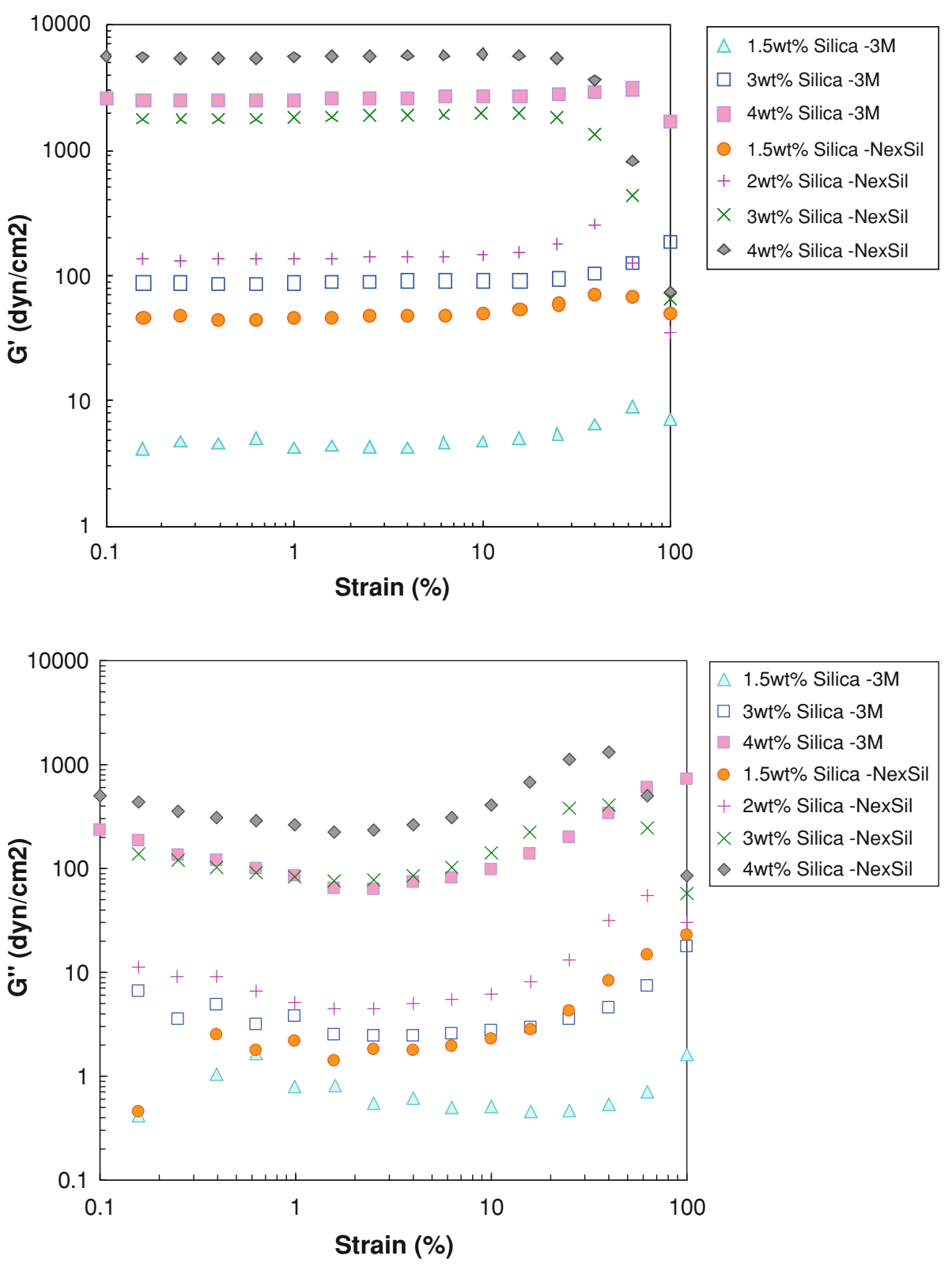


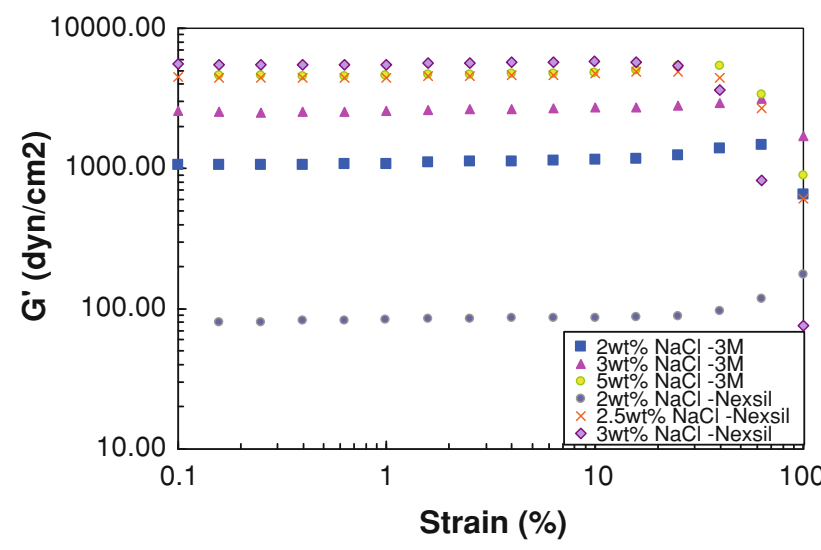

Fig. 13 Storage modulus $\left(G^{\prime}\right)$ as a function of $\mathrm{NaCl}$ concentration at $25{ }^{\circ} \mathrm{C}$

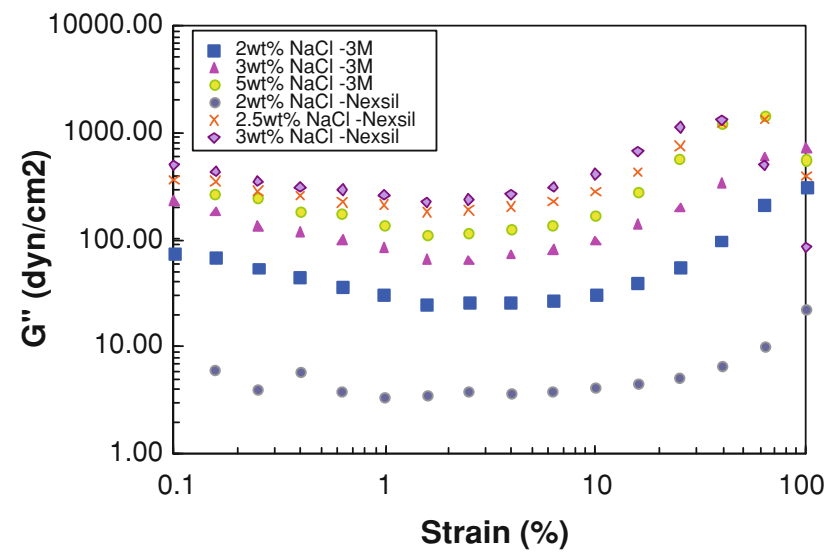

Fig. 14 Loss modulus $\left(G^{\prime \prime}\right)$ as a function of $\mathrm{NaCl}$ concentration at $25{ }^{\circ} \mathrm{C}$

dissipative. Destruction of the structure or breakdown of the filler network occurs at higher strain, and the loss modulus decreases because of further breakdown of the structure (Yziquel et al. 1999). Aggregate size decreases as the silica concentration increases (Metin et al. 2012a). The increase in $G^{\prime \prime}$ as silica concentration increases is likely a result of these smaller, more dissipative aggregates at higher silica concentrations.

Martin and Wilcoxon (1989) discussed that at small strain, Brownian motion is able to restore the structure to the equilibrium value during the oscillation cycle. Therefore, the storage modulus remains constant. After a certain strain, the strain amplitude becomes significant and the Brownian motion is no longer capable of restoring the microstructure. Above this critical strain, the storage modulus decreases, and the loss modulus continues to rise with strain amplitude. This behavior is exhibited in both silica nanoparticle gels studied. The critical strain determined from Figs. 9 and 11 appears to be constant as a

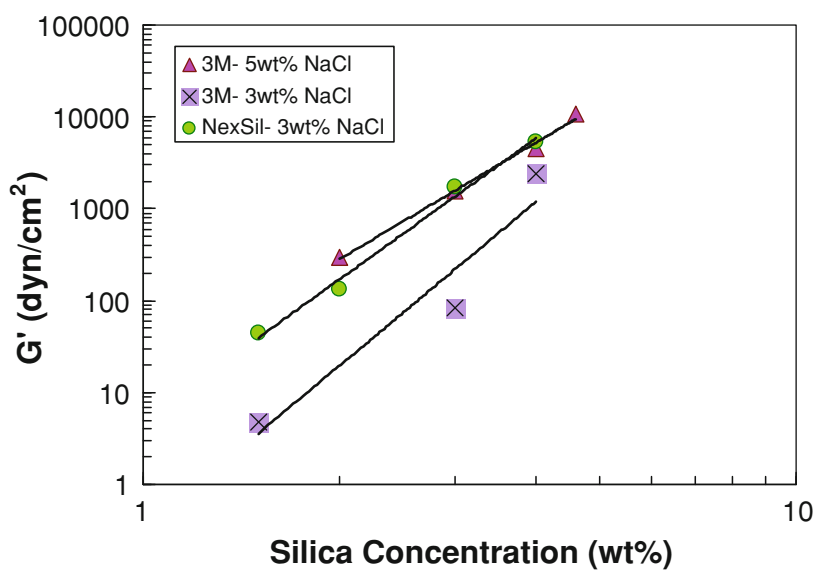

Fig. 15 Storage modulus $\left(G^{\prime}\right)$ as a function of silica concentration at $25{ }^{\circ} \mathrm{C}$. The lines correspond to power law fit



Fig. 16 Storage modulus $\left(G^{\prime}\right)$ as a function of $\mathrm{NaCl}$ concentration at $25{ }^{\circ} \mathrm{C}$

function of silica concentration. The samples withstand a minimum critical strain of $10 \%$ before the structure breaks. On the other hand, the critical strain does not have a clear dependency on salt concentration for both types of particles studied. Below the critical strain, $G^{\prime}$ increases with particle or $\mathrm{NaCl}$ concentration (Figs. 15, 16). This power law behavior was also reported by Shih et al. (1990) with boehmite alumina gels. The authors considered the structure of a gel as a collection of flocs, which are fractal objects closely packed throughout the sample. A continuous network of particles is formed before settling occurs with the resulting suspension having a very high viscosity and a finite shear modulus. The authors observed a power law behavior of $G^{\prime}$ versus $\phi^{m}$. In our experiments, $m$ is calculated to be 5.9 and 4.2 for 3 and 5 wt $\% \mathrm{NaCl} 3 \mathrm{M}$ particles and 5.1 for 3 wt $\% \mathrm{NaCl}$ NexSil5 particles. An increase in $m$ shows that the elasticity increases more rapidly and the network becomes more resistive (Yziquel et al. 1999). 


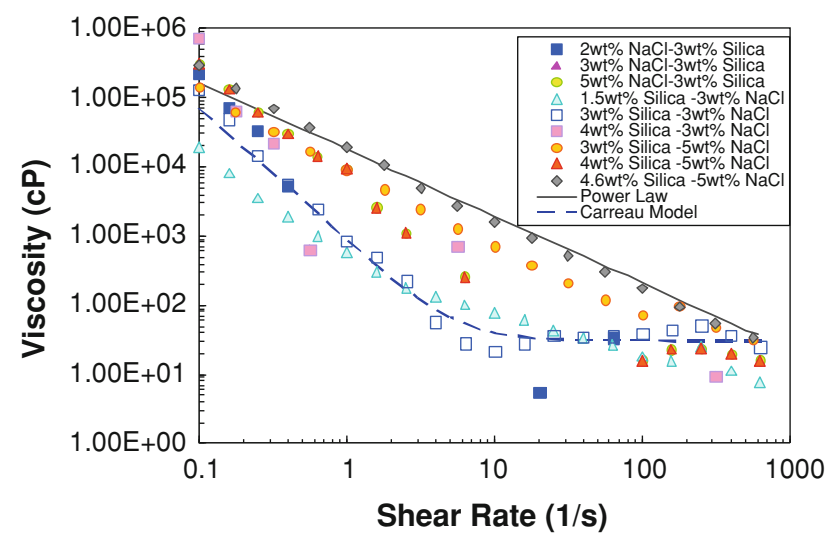

Fig. 17 Viscosity profile for $3 \mathrm{M}$ nanoparticle gels at varying nanoparticle and $\mathrm{NaCl}$ concentrations. The solid line is a power law model fit, and the dashed line is a Carreau model fit

For a solid-like complex fluid, the steady shear stress is independent of shear rate and the shear viscosity, $\eta$, decreases with increasing shear rate, $\dot{\gamma}$, as $\eta \propto \dot{\gamma}^{-1}$. A decreasing shear viscosity with increasing shear rate is called shear thinning (Larson 1999). The viscosity for each gel as a function of steady shear rate was measured $10,000 \mathrm{~s}$ after gelation. All of the gels exhibited shear thinning behavior with $\eta \propto \dot{\gamma}^{-1}$ (see Fig. 17). Most of the samples display a power law decrease in viscosity with increasing shear rate. These samples can be modeled with the power law model (Eq. 3). An example of power law behavior fit to the $4.6 \mathrm{wt} \% 3 \mathrm{M}$ silica, $5 \mathrm{wt} \% \mathrm{NaCl}$ gel is shown as a solid line in Fig. 17. The remaining samples display power law behavior at lower shear rates with a Newtonian plateau at higher rates. These samples, such as 3 wt $\% 3 \mathrm{M}$ silica, 3 wt \% NaCl (dashed line in Fig. 17), can be fit with the Carreau model (Eq. 4). However, the Carreau model predicts a Newtonian plateau at low and high shear rates. Because this lower plateau region is missing from all samples, it is possible that the Carreau model might not be the optimal fit for all data, but it does capture the behavior recorded.

$\eta=K \dot{\gamma}^{n}$

where $K$ and $n$ are empirical parameters.

$\frac{\eta-\eta_{\infty}}{\eta_{0}-\eta_{\infty}}=\frac{1}{\left(1+\left(K_{1} \dot{\gamma}\right)^{2}\right)^{m_{1} / 2}}$

where $\eta_{0}$ and $\eta_{\infty}$ are viscosities at zero and infinity shear rates, respectively, and represent the Newtonian plateau values. Other empirical parameters in Eq. 4 are $K_{1}$ and $m_{1}$. The parameters of the Carreau model fit to the $3 \mathrm{wt} \% 3 \mathrm{M}$ silica, $3 \mathrm{wt} \% \mathrm{NaCl}$ sample are 3,50,000 cp, $30 \mathrm{cp}, 20 \mathrm{~s}$ and 2 for $\eta_{0}, \eta_{\infty}, K_{1}$ and $m_{1}$, respectively. This sample is the only one showing a significant plateau at high shear rates and slope steeper than -1 .

\section{Conclusions}

We studied the phase behavior of silica nanoparticle suspensions as a function of silica and $\mathrm{NaCl}$ concentrations. Monodisperse 3M silica nanoparticles have a wider window of gelation with respect to salinity than polydisperse NexSil5 particles. These phase behavior diagrams will be used in future in situ tests to determine the appropriate silica concentration and salinity gradient between injected fluid and reservoir to induce the desired degree of gelation. The gelation time decreases exponentially as a function of silica, $\mathrm{NaCl}$ concentration, and temperature. This understanding of the gelation kinetics will determine the flow rates necessary to place the silica gel at the desired penetration depth during future experiments.

The storage modulus, $G^{\prime}$, increases with particle concentration following a power law behavior. The critical strain, $10 \%$, is constant for the silica concentrations studied. This implies that for the two types of particles studied, single-phase and two-phase gels can withstand the same strain before the structure breaks. In contrast, the critical strain does not have a clear dependency on salt concentration for either of the particles studied. Steady shear measurements show that silica nanoparticle gels exhibit non-Newtonian, shear thinning behavior which could be described by the power law model or the Carreau model depending on the particle and $\mathrm{NaCl}$ concentration.

Open Access This article is distributed under the terms of the Creative Commons Attribution License which permits any use, distribution, and reproduction in any medium, provided the original author(s) and the source are credited.

\section{References}

Amiri A, Øye G, Sjöblom J (2011) Temperature and pressure effects on stability and gelation properties of silica suspensions. Colloids Surf A 378(1-3):14-21

Burns LD et al (2008) New generation silicate gel system for casing repairs and water shutoff. SPE 113490 presented at SPE/DOE symposium on improved oil recovery, Tulsa, Oklahoma, 20-23 April 2008

Campbell AI et al (2005) Dynamical arrest in attractive colloids: the effect of long-range repulsion. Phys Rev Lett 94(20):20830112083014

Dai C, You Q, Zhao LF, Xiong W (2010) Study and field application of profile control agent in high temperature and high salinity reservoir. SPE 132765-MS presented at Trinidad and tobago energy resources conference, Port of Spain, Trinidad, 27-30 June 2010

de Candia A et al (2005) Colloidal gelation, percolation and structural arrest. Phys A 358:239-248 
Hyun K, Kim SH, Ahn KH, Lee SJ (2002) Large amplitude oscillatory shear as a way to classify the complex fluids. J Non-Newtonian Fluid Mech 107:51-65

Jurinak JJ, LE Summers (1991) Oilfield applications of colloidal silica gel. SPE Prod Eng 6(11):406-412 (SPE-18505-PA)

Larson G (1999) The structure of complex fluids. Oxford university press, New York

Lu PJ et al (2008) Gelation of particles with short-range attraction. Nature 453:499-504

Manley et al (2005) Time-dependent strength of colloidal gels. Phys Rev Lett 95(4):048302-048305

Martin JE, Wilcoxon JP (1989) Spatial correlation and growth in dilute gels. Phys Rev A 39:252-258

Metin CO, Lake LW, Miranda CR, Nguyen QP (2011) Stability of aqueous silica nanoparticle dispersions. J Nanopart Res 13:839-850

Metin CO et al (2012a) Aggregation kinetics and shear rheology of aqueous silica suspensions. J Nanopart Res, submitted

Metin CO, Rankin KM, Nguyen QP (2012b) Phase behavior and rheological characterization of silica nanoparticle gel. Prepr PapAm Chem Soc, Div Pet Chem 57(1), San Diego, March 2012
Senis D, Allain C (1997) Scaling analysis of sediment equilibrium in aggregated colloidal suspensions. Phys Rev E 55:7797-7800

Shih WH et al (1990) Scaling behavior of the elastic properties of colloidal gels. Phys Rev A 42:4772-4779

Silva RF, Vasconcelos WL (1999) Influence of processing variables on the pore structure of silica gels obtained with tetraethylorthosilicate. Mater Res 2:197-200

Smith WE, Zukoski CF (2006) Aggregation and gelation kinetics of fumed silica-ethanol suspensions. J Colloid Int Sci 304:359-369

Stavland A et al (2011) In-depth water diversion using sodium silicate on snorre-factors controlling in-depth placement. SPE 143836 presented at SPE European formation damage conference, Noordwijk, The Netherlands, 7-10 June 2011

Wang GH, Zhang LM (2009) A bio friendly silica gel for insitu protein entrapment: biopolymer-assisted formation and its kinetic mechanism. J Phys Chem 113:2688-2694

Yziquel F, Carreau PJ, Moan M, Tanguy PA (1999) Rheological modeling of concentrated colloidal suspension. J Non-Newtonian Fluid Mech 86(1):133-155 